\title{
Endoscopic Approach via the Minor Duodenal Papilla
}

\author{
Kazuo Inui Junji Yoshino Hironao Miyoshi \\ Department of Internal Medicine, Second Teaching Hospital, Fujita Health University School of Medicine, \\ Nagoya, Japan
}

\section{Key Words}

Endoscopic approach $\cdot$ Minor duodenal papilla $\cdot$ Pancreas

divisum $\cdot$ Pancreatic stent placement $\cdot$ Santorini's duct

\begin{abstract}
Background/Aims: We describe procedures for endoscopic approach via the minor papilla in symptomatic patients with pancreas divisum, pancreatic stones, and stricture of the Santorini's duct, and neoplasms of the minor papilla: endoscopic minor papilla sphincterotomy, endoscopic pancreatic stone removal, endoscopic stent placement in Santorini's duct, and resection of minor papilla neoplasms. Methods: The most important procedural detail was insertion of a guide wire into Santorini's duct via the minor papilla, requiring gentle manipulation of both the cannula and guide wire to avoid subsequent complications such as pancreatitis. $\boldsymbol{R e}$ sults: Minor papilla sphincterotomy was most effective in the treatment of patients with pancreas divisum and associated with acute recurrent pancreatitis; these patients are the best candidates for endoscopic dorsal duct decompression including minor papilla sphincterotomy and stenting. Endoscopic treatment via the minor duodenal papilla was the only useful method for patients whose lesions could not be approached via the major papilla. It is a safe procedure to relieve pain. Long-term results of dorsal ductal stenting were satisfactory in 76 and $90 \%$ of patients (19/25 and 9/10, re-
\end{abstract}

spectively). Conclusion: The high level of endoscopic skills necessary and the small number of patients who need these procedures should limit this approach to select institutions with appropriate endoscopic expertise.

Copyright $\odot 2010$ S. Karger AG, Basel

\section{Introduction}

Endoscopic treatment of diseases of the pancreatobiliary system is usually approached via the major duodenal papilla, e.g. endoscopic sphincterotomy and endoscopic biliary drainage. However, with refinements in endoscopic instruments and procedures, reports of endoscopic treatment via the minor duodenal papilla are increasing. Due to the small size of the orifice of the minor papilla compared with that of the major papilla, it is difficult to inject contrast medium into the main pancreatic duct. However, in patients in whom the approach via the major papilla is difficult, an approach via the minor papilla may be the only alternative. Here we present procedures for the endoscopic approach via the minor papilla: endoscopic minor papilla sphincterotomy (EMPST), endoscopic removal of pancreatic stones, endoscopic stent placement (ESP), and resection of minor papilla neoplasms.

\section{KARGER}

Fax +4161306 1234 E-Mail karger@karger.ch www.karger.com
(ㄷ) 2010 S. Karger AG, Basel

$0253-4886 / 10 / 0272-0153 \$ 26.00 / 0$

Accessible online at:

www.karger.com/dsu
Kazuo Inui, MD

Department of Internal Medicine, Second Teaching Hospital

Fujita Health University School of Medicine, 3-6-10, Otobashi, Nakagawa-ku Nagoya 454-8509 (Japan)

Tel. +81 52321 8171, Fax +81 52323 9886, E-Mail kinui@fujita-hu.ac.jp 


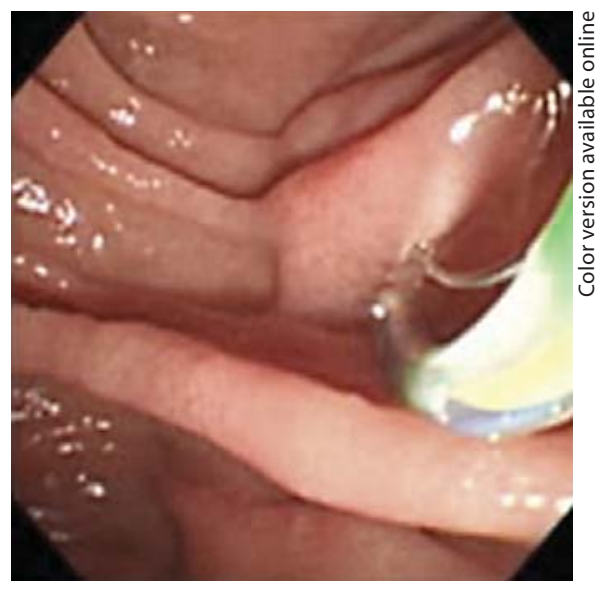

Fig. 1. Endoscopic findings during EMPST with a sphincterotome, briefly pulling slightly towards the 12 to 1 o'clock position.

\section{Endoscopic Minor Papilla Sphincterotomy}

EMPST has been studied by several authors in symptomatic patients with pancreas divisum and associated acute recurrent dorsal pancreatitis [1-3]. In some of our patients with calcific pancreatitis, EMPST preceded endoscopic pancreatic lithotripsy. Endoscopic biopsy was performed via the minor papilla after EMPST for the diagnosis of intraductal papillary mucinous neoplasms of the pancreas [4].

An electroduodenoscope with a biopsy channel 3.7 $\mathrm{mm}$ in diameter (JF-V260; Olympus Medical Systems, Tokyo, Japan) was used, usually with a cannula tapering at the tip (PR-10Q; Olympus Medical Systems) and a guide wire (HydraJagwire; 0.025 inch in diameter, $450 \mathrm{~cm}$ in length; Boston Scientific, Natick, Mass., USA). Occasionally, a metal-tip cannula (PR-24Q-1; Olympus Medical Systems) and a finer guide wire (Jagwire; 0.018 inch in diameter; Boston Scientific) were employed. Minor papilla sphincterotomy was accomplished with a sphincterotome (CleverCut KD-V411M-0720; Olympus Medical Systems, or Autotome RX44; Boston Scientific). An electrosurgical generator (ICC200; ERBE, Tubingen, Germany) facilitated automatically controlled cutting (ENDO Cut). When sphincterotome insertion was difficult, a needle-type sphincterotome was employed (KD-441Q; Olympus Medical Systems). The minor papilla was drained using a 5-french pancreatic stent (Geenen; Cook Medical, Tokyo, Japan) or a nasobiliary drainage catheter (PBD-24Z; Olympus Medical Systems).
Patients were in the prone position on the fluoroscopy table. Premedication consisted of atropine sulfate $(0.5$ $\mathrm{mg}$ ), pentazocine (15 mg), scopolamine butylbromide (2 $\mathrm{mg}$ i.m.), and diazepam (10 $\mathrm{mg}$ i.v.). After insertion of the electroduodenoscope into the second portion of the duodenum, endoscopic retrograde cholangiopancreatography (ERCP) was performed. In patients with a well-developed minor papilla, a guide wire can be easily inserted via the papilla after injection of contrast medium with a tapered-tip cannula. After this injection, a guide wire was inserted through the minor papilla to Santorini's duct. After removal of the cannula, we inserted a sphincterotome alongside the guide wire and cut the minor papilla.

However, the orifice of the minor papilla usually was difficult to find. When contrast medium could not be injected, a metal-tip cannula was employed; in case of failure, a guide wire was directly inserted in combination with a cannula (guide-wire-assisted cannulation). In guide-wire-assisted cannulation, close cooperation between operators manipulating the cannula and those advancing the guide wire was very important, given the need for gentle manipulation of both the cannula and the guide wire to prevent ERCP-induced pancreatitis.

When a direct approach via the minor papilla was still difficult, we tried to insert a guide wire via the major papilla. After insertion of a guide wire into Wirsung's duct and then into the duodenum through Santorini's duct and the minor papilla, we grasped the guide wire and removed it using the biopsy channel of the endoscope. A pancreatic stent or nasobiliary drainage catheter was inserted through the minor papilla into Santorini's duct. These procedures have been reported as the 'rendez-vous method' [5].

After insertion of the guide wire, the cannula was introduced into Santorini's duct. After removal of the cannula, a sphincterotome was advanced along the guide wire into Santorini's duct. We performed EMPST with a sphincterotome, briefly pulling towards the 12 to 1 o'clock position (fig. 1). An incision range was determined within the orifice of the minor papilla, usually $4-6$ $\mathrm{mm}$.

When insertion of the sphincterotome was impossible, we performed a pre-cut minor papilla sphincterotomy with a needle knife beside a previously inserted guide wire, taking care not to cut too much.

Minor papilla sphincterotomy is efficacious in the treatment of pancreatic pain in patients with pancreas divisum $[3,6]$. Patients with acute recurrent pancreatitis benefited more frequently from minor papilla sphincterotomy than those with chronic pancreatitis, so this pro- 
cedure should be avoided in patients with chronic pancreatitis or chronic pancreatic pain [6]. A high success rate of EMPST has been reported (10/11 patients or 91\%) [5].

Complications were noted in 15-29\% of patients with mild pancreatitis $[6,7]$. However, one fatal outcome has been attributed to a pancreatic abscess complicating a failed cannulation [6].

\section{Endoscopic Pancreatic Stone Removal via the Minor Papilla}

Patients with calcified chronic pancreatitis were treated with extracorporeal shockwave lithotripsy [8], and adjuvant endoscopic procedures were performed to remove residual stone fragments from the pancreatic duct. In patients with residual stones in Santorini's duct only or in patients with stricture of the pancreatic duct difficult to approach via Wirsung's duct, pancreatic stones were endoscopically removed via the minor papilla after EMPST. When pancreatic stones were $<5 \mathrm{~mm}$ in diameter, we inserted a basket catheter and removed pancreatic stones through the minor papilla (fig. 2). Stones $>6 \mathrm{~mm}$ in diameter were fragmented to a size $<3 \mathrm{~mm}$ in diameter by extracorporeal shockwave lithotripsy, and then removed by a basket catheter. Only a few previous papers have described EMPST for treating pancreatic stones $[5,9]$.

\section{Endoscopic Stent Placement at the Minor Papilla}

ESP at the minor papilla has been reported by several authors [10-12]. Indications for ESP were pancreas divisum with recurrent pancreatitis, pseudocysts after acute pancreatitis, and pancreatic fistulae with symptoms caused by strictures of the main pancreatic duct resulting in dilation of the tail of the pancreas. In a study by Catalano et al. [10], factors predictive of success were the presence of strictures, pseudocysts $\geq 6 \mathrm{~cm}$, pseudocysts in the body of the pancreas, and pseudocyst duration $<6$ months.

Instruments and methods for cannulation to the minor papilla are the same as in EMPST. After insertion of a guide wire into Santorini's duct via the minor papilla, a 5- or 7-french plastic stent was placed via the guide wire. If stent placement was impossible, a pre-cut accessory sphincterotomy was performed with a needle knife.

Endoscopic Approach via the Minor Duodenal Papilla

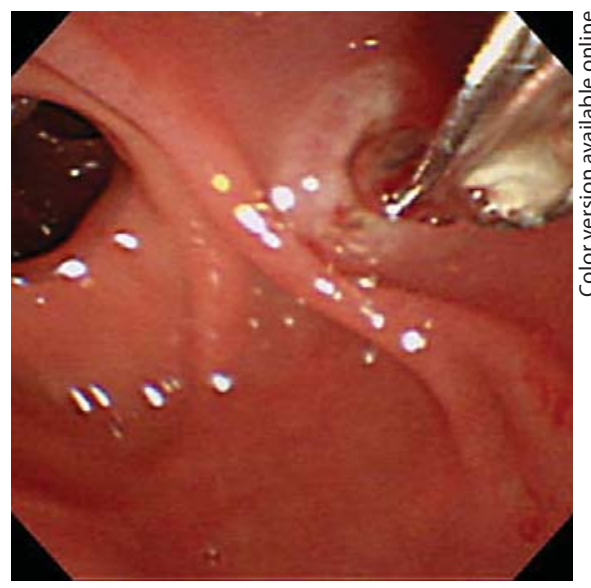

Fig. 2. Endoscopic findings during pancreatic stone removal via the minor papilla after EMPST. A basket catheter grasped a small stone.

Satisfactory long-term results of dorsal ductal stenting have been reported in 76 (19 of 25) and 90\% (9 of 10) of patients [11, 12]. Kamisawa [2] concluded that patients with pancreas divisum associated with acute recurrent pancreatitis are the best candidates for endoscopic procedures including EMPST and stenting.

Reported complications have included stent migration with persistent lodging in the pancreas (2 of 39 stents) [7]. Of 9 patients evaluated, 8 demonstrated new morphologic changes in the ducts on follow-up pancreatograms [7].

\section{Endoscopic Resection of the Minor Papilla}

Recently, endoscopic treatment of adenomas of the major duodenal papilla was reported by several authors [13-15]. Endoscopic major papillectomy using a snare is recognized as a safe, well-tolerated alternative to surgical therapy [16]. Fewer papers [17-19] have described endoscopic minor papillectomy, since tumors of the minor duodenal papilla are rare. Sugiyama et al. [17] reported successful and safe treatment of a sessile adenoma of the minor papilla in a patient without pancreas divisum using endoscopic resection, recommending endoscopic resection and histologic examination of the entire minor papilla adenoma. Additionally, Kanamori et al. [19] performed endoscopic papillectomy in a patient with a minor papilla adenoma associated with pancreas divisum, including pancreatic ESP in the minor papilla to assure drainage after the endoscopic papillectomy.

Dig Surg 2010;27:153-156 
In experienced hands, endoscopic resection of the minor papilla is technically feasible, safe, and associated with favorable clinical outcomes [18], and the complications of open surgery can thus be avoided. Patients should be carefully selected and followed up endoscopically, considering that in $33 \%$ of the patients treated with endoscopic major papillectomy tumor recurrence is observed [20].

\section{Conclusion}

Endoscopic treatment via the minor duodenal papilla is a useful method for patients in whom an approach via the major papilla is impossible. However, early (e.g. postoperative pancreatitis and bleeding) and late complications (e.g. recurrent stricture of the minor papilla or the main pancreatic duct) can still arise. Because high-level endoscopic skills are required and relatively few patients require these procedures, this endoscopic approach to pancreatic diseases should be limited to select institutions having greatest endoscopic expertise.

\section{References}

1 Fukumori D, Ogata K, Ryu S, Maeshiro K, Ikeda S: An endoscopic sphincterotomy of the minor papilla in the management of symptomatic pancreas divisum. Hepatogastroenterology 2007;54:561-563.

$\checkmark 2$ Kamisawa T: Endoscopic approach to the minor duodenal papilla: special emphasis of endoscopic management on pancreas divisum. Dig Endosc 2006;18:252-255.

>3 Borak GD, Romagnuolo J, Alsolaiman M, Holt EW, Cotton PB: Long-term clinical outcomes after endoscopic minor papilla therapy in symptomatic patients with pancreas divisum. Pancreas 2009;38:903-906.

$\checkmark 4$ Watanabe Y, Inui K, Nakazawa S, Yoshino J, Wakabayashi T, Okushima K, Nakamura Y, Nimura Y, Arai T: Nonmucin-producing intraductal papillary adenocarcinoma of the pancreas in a young woman - a case report. Gastrointest Endosc 2001;54:636-639.

$\checkmark 5$ Song MH, Kin MH, Lee SK, Lee SS, Han J, Seo DW, Min YI, Lee DK: Endoscopic minor papilla interventions in patients without pancreas divisum. Gastrointest Endosc 2004;59:901-905.

6 Lehman GA, Sherman S, Nisi R, Hawes RH: Pancreas divisum: results of minor sphincterotomy. Gastrointest Endosc 1993;39:1-8.

7 Cohen SA, Rutkovsky FD, Siegel JH, Kasmin FE: Endoscopic stenting and sphincterotomy of the minor papilla in symptomatic pancreas divisum: results and complications. Diagn Ther Endosc 1995;3:131-139.
-8 Inui K, Tazuma S, Yamaguchi T, Ohara H, Tsuji T, Miyagawa H, Igarashi Y, Nakamura Y, Atomi Y: Treatment of pancreatic stones with extracorporeal shock wave lithotripsy: results of a multicenter survey. Pancreas 2005;30:26-30.

-9 Renzulli P, Müller C, Uhl W, Scheurer U, Buchler MW: Impacted papilla minor stone in pancreas divisum causing severe acute pancreatitis: a case for early ERCP in acute pancreatitis of unknown origin. Digestion 1999;60:281-283.

10 Catalano MF, Geenen JE, Schmalz MJ, Johnson GK, Dean RS, Hogan WJ: Treatment of pancreatic pseudocysts with ductal communication by transpapillary pancreatic duct endoprosthesis. Gastrointest Endosc 1995; 42:214-218.

-11 Lans JI, Geenen JE, Johanson JF, Johnson GK, Dean RS, Hogan WJ: Endoscopic therapy in patients with pancreas divisum and acute pancreatitis - a prospective, randomized, controlled clinical trial. Gastrointest Endosc 1992;38:430-434.

12 Ertan A: Long-term results after endoscopic pancreatic stent placement without pancreatic papillotomy in acute recurrent pancreatitis due to pancreatic divisum. Gastrointest Endosc 2000;52:9-14.

13 Binmoeller KF, Boaventura S, Ramsperger K, Soehendra N: Endoscopic snare excision of benign adenomas of the papilla of Vater. Gastrointest Endosc 1993;39:127-131.
14 Catalano MF, Linder JD, Chak A, Sivak MV Jr, Raijman I, Geenen JE, Howell DA: Endoscopic management of adenoma of the major duodenal papilla. Gastrointest Endosc 2004; 59:225-232.

$>15$ Ito K, Fujita N, Noda Y, Kobayashi G, Horaguchi J, Takasawa O, Obana T: Preoperative evaluation of ampullary resection with EUS and transpapillary intraductal US: a prospective and histopathologically controlled study. Gastrointest Endosc 2007;66:740-747.

-16 Pandolfi M, Martino M, Gabbrielli A: Endoscopic treatment of ampullary adenomas. JOP 2008;9:1-8.

17 Sugiyama M, Kimura W, Muto T, Yahagi N, Ichinose M, Miki K: Endoscopic resection of adenoma of the minor papilla. Hepatogastroenterology 1999;46:189-192.

18 Trevino JM, Wilcox CM, Varadarajulu S: Endoscopic resection of minor papilla adenomas (with video). Gastrointest Endosc 2008; 68:383-386.

19 Kanamori A, Kumada T, Kiriyama S, Sone Y, Tanikawa M, Hisanaga Y, Toyoda H, Kawashima $\mathrm{H}$, Itoh A, Hirooka Y, Goto H: Endoscopic papillectomy of minor papilla adenoma associated with pancreas divisum. World J Gastroenterol 2009; 15:1138-1140.

20 Cheng CL, Sherman S, Fogel EL, Howard TJ, Lazzell-Pannell L, Lehman GA: Endoscopic snare papillectomy for tumors of the duodenal papillae. Gastrointest Endosc 2004;60: 757-764. 